\title{
ARTICLE
}

Cite this: DOI: 10.1039/xoxxooooox

\section{Dual mode quantitative imaging of microscopic viscosity using a conjugated porphyrin dimer†}

\author{
Aurimas Vyšniauskas, ${ }^{a}$ Milan Balaz, ${ }^{b} \ddagger$ Harry L. Anderson ${ }^{b}$ and Marina K \\ Kuimova ${ }^{a}$ *
}

Received ooth January 2012,

Accepted ooth January 2012

DOI: $10.1039 /$ xoxxooooox

www.rsc.org/

\begin{abstract}
Microviscosity is of paramount importance in materials and bio-sciences. Fluorescence imaging using molecular rotors has emerged as a versatile tool to measure microviscosity, either using a fluorescence lifetime or a ratiometric signal of the rotor; however, only a limited number of blue-to-green-emitting fluorophores with both the lifetime and the ratiometric signal sensitivity to viscosity have been reported to date. Here we report a deep red emitting dual viscosity sensor, which allows both the ratiometric and the lifetime imaging of viscosity. We study viscosity in a range of lipid-based systems and conclude that in complex dynamic systems dual detection is preferable in order to independently verify the results of the measurements as well as perform rapid detection of changing viscosity.
\end{abstract}

\section{Introduction}

Viscosity is an important characteristic of a microenvironment, since it determines the micro- and macroscopic properties of materials and controls the rates of processes and reactions on the microscopic scale. The need to detect microscopic viscosity is widely recognised in biochemical and biological research, ${ }^{1}$ and new applications are emerging in material and atmospheric sciences. $^{2-4}$ Among several methods that allow the detection of viscosity on a microscopic scale, such as fluorescence correlation spectroscopy (FCS) ${ }^{5,6}$ fluorescence recovery after photobleaching (FRAP), ${ }^{7,8}$ single particle tracking (SPT), ${ }^{9,10}$ steady state and time-resolved fluorescence anisotropy, ${ }^{11-13}$ detection fluorescence of molecular rotors has secured its place as a versatile technique that allows non-destructive imaging of viscosity in heterogeneous samples and on-line detection of changing viscosity. These two advantages are not accessible simultaneously with any other existing technique. ${ }^{14,15}$ In molecular rotors, the fluorescence competes with intramolecular rotation, which is viscosity dependent. As a consequence, the fluorescence intensity and the fluorescence decay of the excited state are strongly affected by the viscosity of the microenvironment of the probe.

It is now well established that quantitative imaging of viscosity requires either ratiometric detection or fluorescence lifetime measurements, in order to rule out the effect of the concentration of the probe on the observed signal. ${ }^{16-19}$ Consequently, both types of molecular rotors, using either ratiometric or lifetime detection, have been reported and used for imaging in a variety of systems, from live cells to lipid membranes to atmospheric aerosols..$^{2,16-18,20}$ Here we report a red emitting molecular rotor 1, Figure 1a, previously synthesized by Balaz et. al. ${ }^{21}$ but not explored as a molecular rotor until now. We have discovered that $\mathbf{1}$ can be used in both modalities of viscosity imaging: lifetime and ratiometric. Only a few dual-mode molecular rotors have been published to date, combining the capabilities of both these approaches. ${ }^{18,22,23}$ However, their limited dynamic range for ratiometric measurements complicated the reliable and direct comparison of both techniques in heterogeneous samples. In addition, the blue shifted excitation and emission spectra of these rotors, which contain bands in 400-500 $\mathrm{nm}$ range, make them less useful for in vivo experiments.

Molecular rotor 1 emits in the red region of the spectrum (640$710 \mathrm{~nm}$, Figure 1), which sets it apart from most existing molecular rotors published to date. In this work we use $\mathbf{1}$ for measuring viscosity in lipid monolayers, bilayers and in cells. We use these systems to highlight the advantages and limitations of both ratiometric and lifetime approaches. Based on the data for $\mathbf{1}$ we demonstrate why it might be necessary to have both modalities of quantitative imaging available.

\section{Methods}

\section{Calibration of 1}

Dimer 1 was synthesised as previously described. ${ }^{21}$ A $1 \mathrm{mM}$ solution of the porphyrin dimer in dimethylsulfoxide (DMSO) was used as a stock solution. The viscosity calibration was performed in methanol and glycerol mixtures at room temperature, covering a viscosity range between 0.6 and 1458 cP. Viscosities of the mixtures were measured using Stabinger 
viscometer (SVM3000, Anton Paar). All calibration mixtures contained $\sim 0.1 \%(v / v)$ of DMSO due to using the stock solution of the dye in DMSO. We have confirmed that at such a low concentration DMSO did not affect viscosity measurements. Additionally, DMSO is fully miscible with methanol/glycerol mixtures, which allowed us to rule out the preferential solvation of the dye by DMSO as a potential source of artefacts. Methanol and glycerol were spectroscopic grade obtained from Sigma-Aldrich. Dye solutions for time-resolved fluorescence experiments were prepared by dissolving the required amount of stock solution in a relevant methanol/glycerol mixture and sonicating the solution for two minutes at $40{ }^{\circ} \mathrm{C}$ to aid full solubilisation of $\mathbf{1}$. The final concentration of porphyrin dimer for these measurements was $1.33 \mu \mathrm{M}$.

Fluorescence decays were measured using Fluorescence Lifetime Imaging Microscopy (FLIM) setup consisting of a Leica SP 5 II confocal laser scanning microscope; a Coherent Chameleon Vision II mode-locked femtosecond Ti:sapphire laser and a Becker \& Hickl SPC-830 (time-correlated single photon counting) TCSPC card. The laser was operating at 80 $\mathrm{MHz}$ and producing $140 \mathrm{fs}$ pulses. The output wavelength was tuneable between $680 \mathrm{~nm}$ and $1080 \mathrm{~nm}$. The required laser wavelength for one-photon excited fluorescence was obtained by frequency doubling the output of the Ti:sapphire laser with second harmonic generation crystal (SHG, Harmonic, Coherent). $453 \mathrm{~nm}$ light was used for exciting the dimer 1, in order to obtain preferential absorption by the twisted conformer. $\mathrm{x} 20, \mathrm{x} 40$ and $\mathrm{x} 63$ objectives were used for measuring the emission of $\mathbf{1}$ in lipid monolayers, giant unilamellar vesicles (GUVs) and SK-OV-3 cells, respectively. The confocal pinhole was fully opened in order to collect the maximum signal. Fluorescent light was emitted over $600 \mathrm{~nm}-$ $750 \mathrm{~nm}$ range and detected with a photomultiplier tube (PMC100-1, Hamamatsu). The light of the desired wavelength was detected by dispersing all emitted light through the prism and changing the width and the position of slits in front of the PMT. 1024 time bins were used for acquiring fluorescence decays. Acquisition times were chosen such that the decays had approximately 10000 counts at the peak. The instrument response function (IRF) was obtained by recording the scattering curve from a glass coverslip. Dye solutions were kept in Lab-Tek (Nunc, Thermo Scientific) chamber slides during measurements.

\section{Preparation of lipid monolayers}

Stock solutions of 1,2-dioleoyl-sn-glycero-3-phosphocholine (DOPC) and 1,2-diphytanoyl-sn-glycero-3-phosphocholine (DPhPC) in chloroform were obtained from Avanti Polar Lipids. A procedure for the preparation of lipid monolayers was adopted from Holden et al. ${ }^{24}$ Lipid solutions in dodecane were prepared by evaporating chloroform from the required amount of lipid stock solution and then dissolving resulting lipid film in dodecane in order to obtain the final concentration of lipid of 1 $\mathrm{mg} / \mathrm{mL}$. Then the stock solution of $\mathbf{1}$ in DMSO was added, followed by sonication for $5 \mathrm{~min}$ at $40{ }^{\circ} \mathrm{C}$. The concentration of 1 was $3 \mu \mathrm{M}$. $60 \mu \mathrm{L}$ of $0.11 \mathrm{M} \mathrm{KCl}$ solution in water was then

added into $500 \mu \mathrm{L}$ lipid and dye mixture in dodecane and the vial was shaken by hand and left to stand for $15 \mathrm{~min}$. Finally, the resulting mixture of water droplets coated in lipid monolayer in dodecane was transferred onto depression slide and imaged.

\section{Preparation of Large Unilamellar Vesicles (LUVs)}

Dry lipid-dye film was prepared by evaporating chloroform solution of lipid and 1 mixed at 1000:1 lipid-to-dye ratio. Water was added and the vial was vortexed in order to obtain a uniform suspension. Lipid concentration in the suspension was $1 \mathrm{mg} / \mathrm{mL}$. LUVs were prepared from the suspension by continuously passing the heated suspension at $60{ }^{\circ} \mathrm{C}$ through Whatman nucleopore track-etched membrane with $200 \mathrm{~nm}$ pores using mini-extruder from Avanti Polar Lipids (at least 10 passes).

\section{Preparation of Giant Unilamellar Vesicles (GUVs)}

GUVs were prepared by the electroformation method. ${ }^{25}$ First, 2 $\mu \mathrm{L}$ of $10 \mathrm{mg} / \mathrm{mL}$ lipid and $\mathbf{1}$ solution in chloroform was spread on ITO (Indium-Tin Oxide) slide on $1 \mathrm{~cm}^{2}$ area and evaporated under $\sim 2 \mathrm{mPa}$ pressure for $30 \mathrm{~min}$. Lipid-to-dye ratio was 1000 . After evaporation, electroformation chamber was assembled from two ITO slides and polydimethylsiloxane (PDMS) spacer, filled with $200 \mathrm{mM}$ sucrose solution in water and connected to the TTi TG550 function generator. Finally, 1.2 V alternating voltage at $10 \mathrm{~Hz}$ frequency was applied and the chamber was kept in the oven at $60{ }^{\circ} \mathrm{C}$ temperature for $1.5 \mathrm{~h}$. The resulting GUVs were transferred into $200 \mathrm{mM}$ glucose solution in water and imaged in a Lab-Tek chamber slide.

\section{Acquisition of fluorescence lifetime images, ratiometric images and fluorescence spectra}

FLIM, ratiometric images and fluorescence spectra were all collected using the Leica SPII microscope setup described above. Samples were excited at $453 \mathrm{~nm}$. Fluorescence decays were detected between 620 and $750 \mathrm{~nm}$ and $635-645 \mathrm{~nm}$ decays were used for calibration as described in the Results and Discussion section. FLIM images in lipid monolayers and in GUVs were detected at $650 \mathrm{~nm}-660 \mathrm{~nm}$ to account for a 15 $\mathrm{nm}$ red solvatochromic shift of the fluorescence spectrum of $\mathbf{1}$ (see Figure S5). FLIM images were acquired at 256x256 pixel resolution using 256 time bins. The scanning frequency was $400 \mathrm{~Hz}$. Ratiometric calibration was performed by dividing two fluorescence images obtained simultaneously using two detectors: 635-645 nm (Detector 1) and 690-700 nm (Detector $2)$. Ratiometric images of lipid monolayers, GUVs and cells were obtained using $650-660 \mathrm{~nm}$ and 705-715 $\mathrm{nm}$ windows to account for the $15 \mathrm{~nm}$ red shift of the spectra. Fluorescence spectra of $10 \mathrm{~nm}$ resolution were obtained using the microscope setup by sliding detection window of $10 \mathrm{~nm}$ width along wavelength axis by $5 \mathrm{~nm}$ and measuring mean fluorescence intensity $(\lambda$-scan).

\section{Cell work}


SK-OV-3 human ovarian carcinoma cell line was used for cell imaging experiments. Cells were cultured in Dulbecco's modified Eagle's medium (DMEM) with 10\% foetal bovine serum (FBS) and split when $\sim 70 \%$ confluent. $5 \% \mathrm{CO}_{2}$ and $37^{\circ} \mathrm{C}$ was used for incubating cells. Cells were seeded into 8well Lab-Tek chamber for imaging by transferring 50000 cells per well and allowing them to grow for 24 hours. $10 \mu \mathrm{M}$ solution of 1 in DMEM with $10 \%$ FBS and 1\% DMSO was used for staining cells. The cell staining solution was first passed through a $200 \mathrm{~nm}$ filter to remove aggregates and warmed in the incubator at $37^{\circ} \mathrm{C}$ for $30 \mathrm{~min}$ before administering to the cells. The cells were incubated for $24 \mathrm{~h}$ and washed 3 times with Hank's Balanced Salt Solution (HBSS) before imaging at $37^{\circ} \mathrm{C}$.

\section{Data analysis}

FLIM images were fitted and analysed using FLIMfit software tool developed at Imperial College London (v4.6.1) ${ }^{26}$ and Becker \& Hick1 SPCImage v3.9.7 software. Pixels were binned in order to get around 1000 counts at the peak of each decay. Multiexponential fitting for single decays traces (for the calibration), data processing and analysis was done in MATLAB R2012a and OriginPro 8.6.

\section{Results and Discussion}

Butadiyne linked porphyrin dimer $\mathbf{2}$ was previously shown to have molecular rotor properties, via a ratiometric detection method. ${ }^{17}$ The working principle of this type of molecular rotor is based on the fact that two fluorescent conformers of a dimer exist, with twisted and planar position of the porphyrin rings, which can interconvert with viscosity dependent rates. ${ }^{27}$ Here we tested the molecular rotor properties of $\mathbf{1}$, a dimer analogous to 2 , with excellent biocompatibility properties. ${ }^{28}$

The absorption and fluorescence spectra of $\mathbf{1}$ along with the proposed energy levels diagram are shown in Figure 1b,c. The excitation into higher energy absorption bands (depicted by green dots in either $\mathrm{Q}$ or Soret bands) results in the excitation of a twisted conformer, ${ }^{29}$ which can either emit light $(640 \mathrm{~nm}$, green dot on the insert) or interconvert into a planar form, which emits at $700 \mathrm{~nm}$ (red dot on the insert).

The twisted to planar conformer conversion is viscositydependent and thus the ratio of the two bands: 640 vs $695 \mathrm{~nm}$, can be calibrated against the solution viscosity (Figure 2c), using the Förster-Hoffmann equation. ${ }^{14,30}$

Importantly, the viscosity sensitivity can only be achieved by exciting the dimer preferentially in the twisted conformation (blue arrow in Figure 1c), e.g. at ca $450 \mathrm{~nm}$ or $630 \mathrm{~nm}$ wavelengths for 1 . Due to the limitations of our experimental setup, in this work we used $453 \mathrm{~nm}$ pulsed excitation to prepare the viscosity-sensitive excited state of $\mathbf{1}$, however, it is also possible to use the pulsed excitation at $630 \mathrm{~nm},{ }^{29}$ which efficiently excites the twisted conformer of $\mathbf{1}$ and, at the same time, takes full advantage of its red shifted absorption and emission bands that show good overlap with the so-called 'tissue optical window'.
The viscosity-dependent intensity ratio 640:695 $\mathrm{nm}$ ranges from 0.05 to 1.6 , providing an extremely large dynamic range for ratiometric measurements of viscosity between 1 and 1000 cP. Additionally, both emission bands of the dimer lie in the red region of the spectrum, in the tissue optical window, providing optimal tissue penetration. These two factors set the dimer apart from all other ratiometric rotors available to date and offer significant advantages during live cell and tissue imaging.

Ratiometric viscosity measurements can offer concentrationindependent viscosity data as well as extremely fast image recording times; however, they might suffer from artefacts due to environmentally induced shifts in fluorescence or absorption spectra as well as from binding of the probe. On the other hand, the fluorescence lifetime detection method is capable of distinguishing between true viscosity measurements and artefacts, by detecting individual fluorescence decays from all species in the detection volume, thus reporting on undesirable events such as binding or quenching, if present, which reveal themselves as additional exponential component in fluorescence decay. It follows that dual probes, providing advantages of both the ratiometric and the lifetime detection methods, are highly desirable.

Given the interplay of conformer energies shown in Figure 1c we hypothesised that the fluorescence lifetime of the twisted form of $\mathbf{1}$ is likely to display strong viscosity dependence, due to a twisting originating from this state. We have recorded a series of fluorescence decay traces between $630-730 \mathrm{~nm}$, in methanol/glycerol mixtures of varied viscosity.
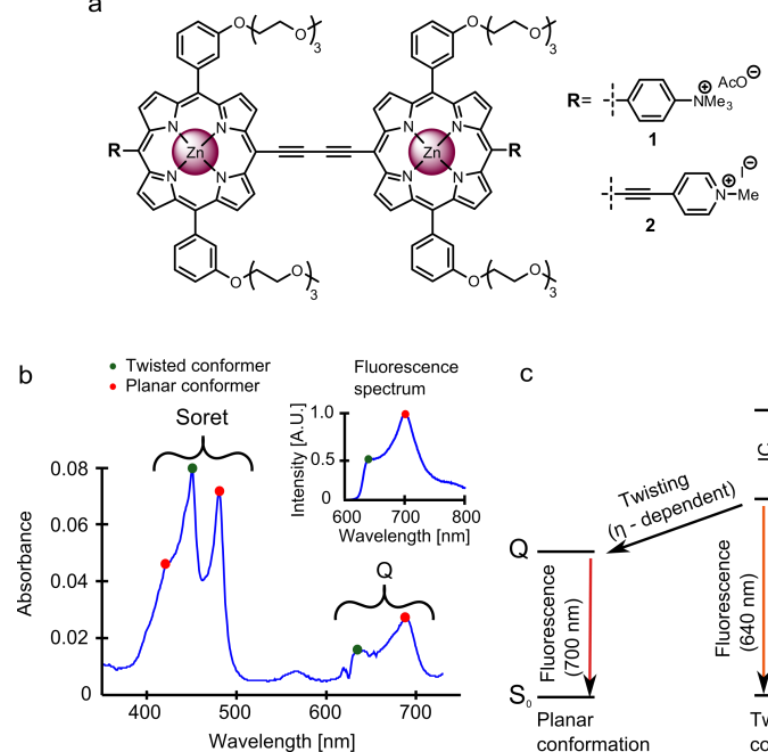

C

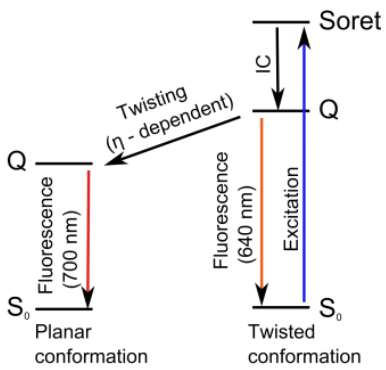

Figure 1. (a) The structure of conjugated porphyrin dimers 1 and 2. (b) An absorption spectrum of 1 recorded in 6:4 glycerol/methanol mixture and a fluorescence spectrum (insert, $\lambda_{\text {exc }}=453 \mathrm{~nm}$ ). Green and red dots indicate absorption bands originating from twisted and planar conformers, respectively, in either $\mathrm{Q}$ or Soret bands. The viscosity-sensing twisted conformer can be excited in either the Soret $(453 \mathrm{~nm})$ or the $Q(630 \mathrm{~nm})$ bands. (c) Energy diagram of conformers of $\mathbf{1}$. IC - internal conversion. 
a

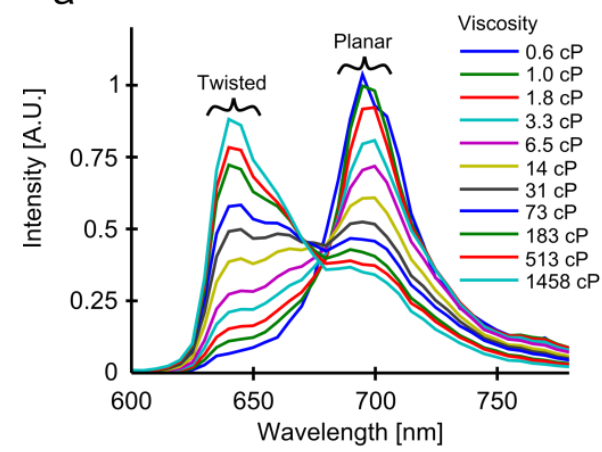

b

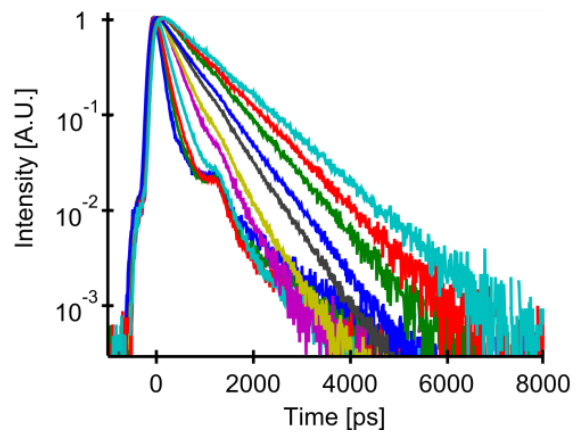

C

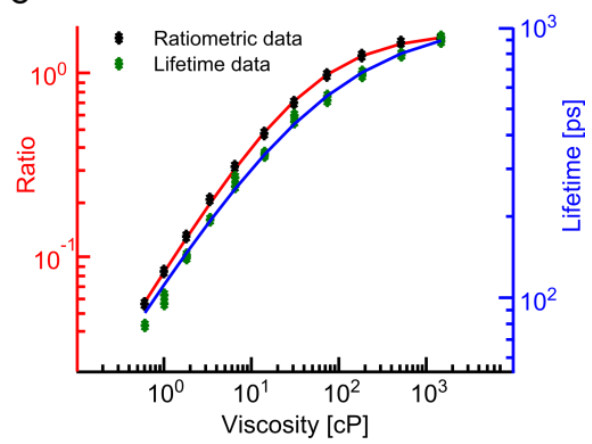

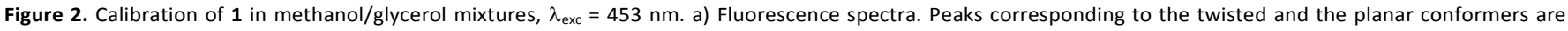
marked. b) Fluorescence decays recorded at $640 \pm 5 \mathrm{~nm}$. c) Ratiometric (red) and lifetime (blue) calibration curves.

The representative decays recorded at $640 \mathrm{~nm}$ are shown in Figure $2 b$; the remaining datasets are shown in the Supporting Information (SI), Figure S1. It is clear from this data that at wavelengths below $700 \mathrm{~nm}$ the decay traces display a very strong sensitivity to viscosity, with lifetimes between 50 ps and $900 \mathrm{ps}$, corresponding to a viscosity range $0.6-1500 \mathrm{cP}$. At the same time, fluorescence decays at and above $700 \mathrm{~nm}$ show minimal viscosity dependence, with a decay constant of 900$1000 \mathrm{ps}$ for all viscosities. In the decays above $700 \mathrm{~nm}$ the increasing viscosity is manifested by an increase in the decay rise time that varies from 50 to ca 900 ps, matching the decay constants recorded at $640 \mathrm{~nm}$ (SI, Figure S2). This match further confirms our proposed photophysical scheme for $\mathbf{1}$, giving evidence that the planar conformer does indeed form predominantly from the twisted form. Here it should be noted that, according to the absorption spectra Figure 1b, some proportion of the planar conformer might be directly excited by $450 \mathrm{~nm}$ light and its emission spectrum overlaps slightly with the detection window at $640 \mathrm{~nm}$. This results in a biexponential decay (Figure 2b) with a small contribution of the planar decay (<5\% amplitude, SI, Figure S2), consistently observed at 640 $\mathrm{nm}$. The calibration graphs produced for $\mathbf{1}$, from both the ratiometric and the lifetime data, Figure. 2c, cover a large dynamic range and conform to the Förster-Hoffmann equation, up to viscosities of ca $800 \mathrm{cP}$, above which the linearity is lost.

Given that porphyrin dimers such as $\mathbf{1}$ have an extremely large two photon absorption cross section, up to $2 \times 10^{5} \mathrm{GM},{ }^{28}$ we performed the ratiometric and lifetime calibration of $\mathbf{1}$ following two photon excitation, varied between 850 and 950 nm (SI, Figure S3). However, due to poor absorption of two photon light by the twisted conformer ${ }^{31}$ because of a loss of conjugation and symmetry, the dynamic range observed in both imaging modalities was significantly reduced compared to the one photon excitation and cannot be considered useful for imaging.

Next, we used $\mathbf{1}$ for dual mode microviscosity measurements in monolayers at a water/oil interface. Initially $\mathbf{1}$ was incorporated into a diphytanoylphosphatidylcholine (DPhPC) lipid monolayer surrounding the water droplet in dodecane. DPhPC was chosen as a saturated lipid that exists in liquid-disordered $\left(\mathrm{L}_{\mathrm{d}}\right)$ phase at room temperature. ${ }^{32}$ Fluorescence of $\mathbf{1}$ was observed only from the dodecane and water interface (SI, Figure S4), confirming the preference of $\mathbf{1}$ for the lipid phase and/or fluorescence enhancement of $\mathbf{1}$ in the presence of a more viscous lipid phase. FLIM and ratiometric images were measured, which yielded viscosity maps shown in Figure 3a. Viscosity values were calculated in each pixel using the calibration curves shown in Figure 2c. Unexpectedly, these two methods gave different viscosity values in the monolayer, which were $3.8 \mathrm{cP}$ and $13.3 \mathrm{cP}$ obtained by ratiometric and lifetime imaging, respectively.

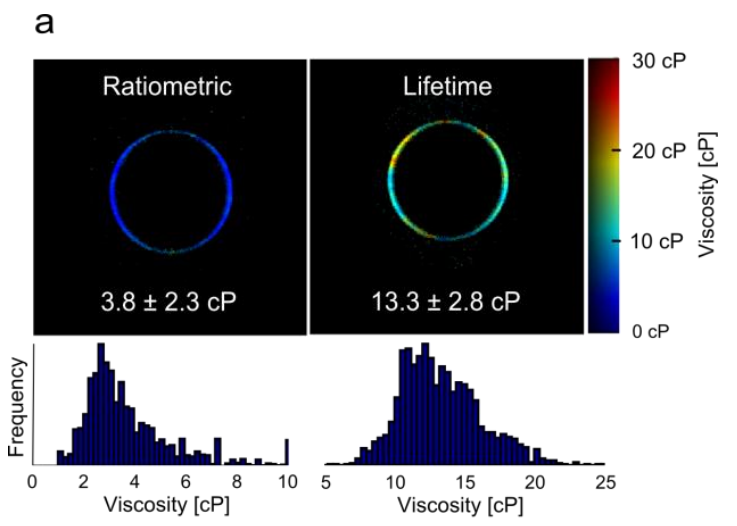

b

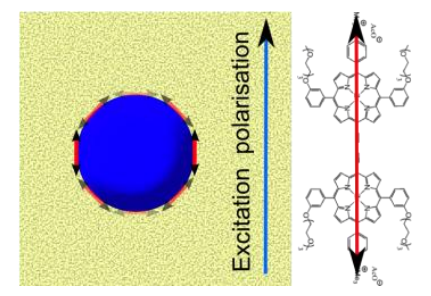

Figure 3. Imaging of $\mathbf{1}$ in DPhPC lipid monolayers. a) Viscosity maps of DPhPC monolayer at the water/dodecane interface obtained by ratiometric (left) and lifetime (right) methods superimposed on intensity images; $\lambda_{\text {exc }}=453 \mathrm{~nm}$ Average viscosity values with the error equal to $1 \sigma$ and distribution histograms are also shown. b) The schematic showing the relationship between the direction of polarisation of excitation light (blue arrow) and arrangement of the dimer molecules at the interface, resulting in the intensity pattern observed in a). The direction of the transition dipole moment for excitation of the dimer is also shown. 
It should be noted that the dominant decay component in FLIM images (responsible for sensing the viscosity) had an amplitude of between $85-90 \%$ in monolayers, compared to a $95-100 \%$ amplitude recorded in calibration mixtures. The other (longer) decay component was previously assigned to a planar conformer ( $5 \%$ in methanol/glycerol mixtures), and these results indicate that there is a preference for the planar conformation of $\mathbf{1}$ in lipid monolayer, stronger than prescribed by solution viscosity alone.

To investigate this further we analysed the uneven intensity distribution of the fluorescence of $\mathbf{1}$ around the water droplet, as shown in Figure 3a: less intense on the top and the bottom and more intense on the sides, while the polarisation of the excitation light was in the image plane as shown by the blue arrow in Figure 3b. These experiments strongly indicate that in Figure $3 \mathrm{a}$ the absorption transition dipole moment of $\mathbf{1}$ is close to being parallel to the polarisation of excitation light at the sides of the droplet (resulting in bright fluorescence) and close to being perpendicular on the top and the bottom of the droplet (resulting in dim fluorescence), Figure 3b. Thus, unexpectedly, 1 preferentially orientates parallel to the water and dodecane interface rather than inserts vertically into the lipid layer. The $15 \mathrm{~nm}$ solvatochromic shift of $\mathbf{1}$ observed in a monolayer compared to methanol/glycerol mixtures (Fig S5, SI), is further confirmation that $\mathbf{1}$ preferentially localises close to an aqueous environment because the fluorescence spectrum of $\mathbf{1}$ in water is red-shifted by $\sim 30 \mathrm{~nm}$. Taken together, these results indicate that 1 localises in the lipid head area, close to the aqueous interface, which is likely to favour a planar conformation of a dimer. Since the proportion of the planar form is higher than what can be expected from the viscosity of the environment, the ratiometric method strongly underestimated the viscosity. The lifetime method, however, is not affected and produces a correct viscosity value based on the fluorescence decay fitting. This example clearly shows that care should be taken when quantitatively interpreting ratiometric data.

We have also prepared lipid bilayers as giant unilamellar vesicles (GUVs) and imaged their viscosity by ratiometric and FLIM approaches. In the case of bilayers, $\mathbf{1}$ demonstrates an increase in viscosity compared to monolayers, by both the ratiometric and the lifetime data (Figure 4). Additionally, 1 reports the same solvatochromic shift as in a monolayer (Figure 4 ), indicating the same or similar localization of the dye. Thus, even though the dimer is not fully incorporated in the lipid tail region of the bilayer, it senses an increased rigidity of the bilayer structure. Both values obtained with the dimer in the monolayers $(13 \mathrm{cP})$ and in the bilayer $(\sim 17 \mathrm{cP})$, are significantly different to those obtained with the fully imbedded lipid probe, Bodipy, which gave viscosity values of $90 \mathrm{cP}$ (SI, Figure S6) and $160 \mathrm{cP}$ in monolayers and bilayer, respectively. ${ }^{33}$ These values are consistent with the lower head region viscosity of a lipid bilayer. ${ }^{34}$
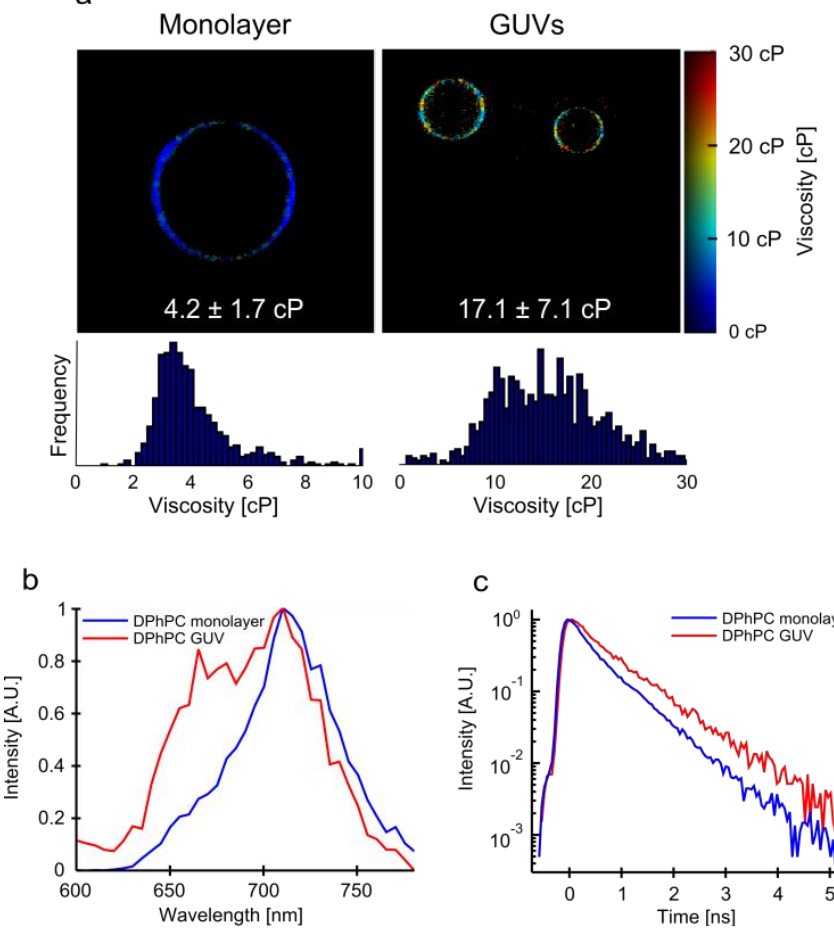

C



Figure 4. (a) The ratiometric viscosity maps of monolayers and GUVs with corresponding distribution histograms. (b) Fluorescence spectra and (c) fluorescence decays of $\mathbf{1}$ in DPhPC lipid monolayer (blue) and GUVs (red). In all three cases, 1 measures considerably higher viscosities in GUVs compared to a monolayer, consistent with a more tight packing of lipids in this case. In (c) lifetimes of the twisted conformer correspond to $14 \mathrm{cP}$ and to $17 \mathrm{cP}$ in monolayer and GUVs, respectively.

To utilise the capability for fast image recording available with a ratiometric method, we have tested the effect of singlet oxygen production by the dimer on the viscosity of a monolayer containing an unsaturated lipid, 1,2-dioleoyl-sn-glycero-3phosphocholine DOPC. Dimer 1 was previously shown to produce singlet oxygen in high yield $\left(\phi_{\Delta}\right.$ determined as 0.7 in methanol, ${ }^{28}$ although it is expected to be dependent on the excitation wavelength and viscosity and was determined to be as low as 0.2 for the wavelengths directly exciting the twisted conformer in viscous media ${ }^{29}$ ). Moreover, we have previously demonstrated, using a ratiometric method and a dimer $\mathbf{2}$ that the viscosity in a single cell increased dramatically during irradiation of the dimer leading to cell death. ${ }^{17}$ The results of an irradiation of a DOPC monolayer containing $\mathbf{1}$ are shown in Figure $5 \mathrm{a}$ on the left, and the average viscosity values obtained from these images in Figure 5b. A large viscosity increase of 6 fold is observed, from 4 to $24 \mathrm{cP}$ (ratiometric), which was almost completely supressed in the presence of $0.11 \mathrm{M} \mathrm{NaN}_{3}$, an efficient singlet oxygen quencher (Figure 5a middle). No increase in viscosity was observed in DPhPC without $\mathrm{NaN}_{3}$ (Figure 5a, right). 


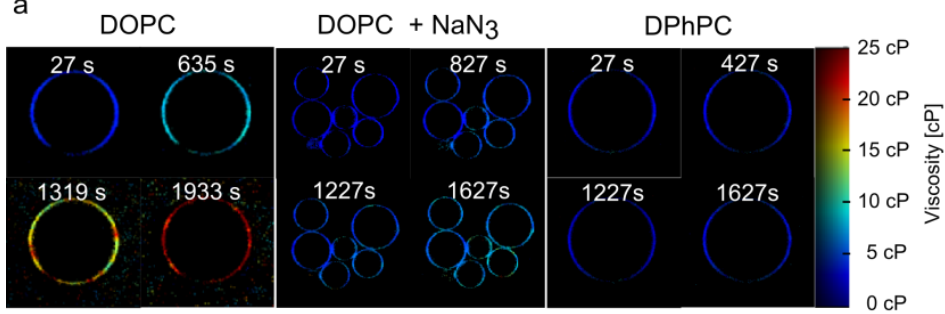

b

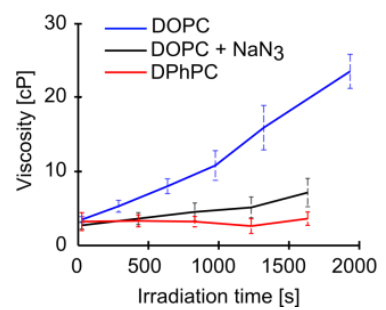

C

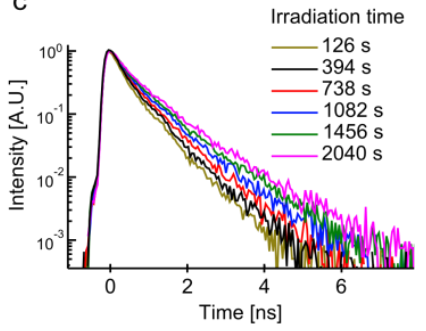

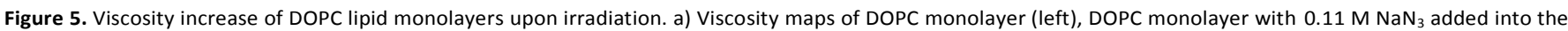

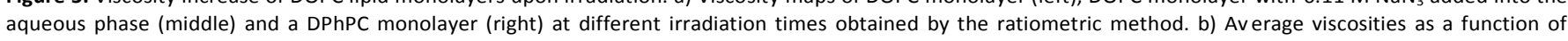

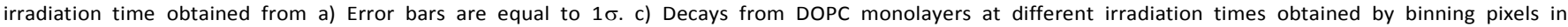
corresponding FLIM images.

In order to analyse the lifetime data, it was required that all decays were summed to produce a good $\mathrm{S} / \mathrm{N}$ ratio, Figure $5 \mathrm{c}$. The decays are biexponential and show increasing average lifetime with irradiation time (Figure 5c). Based on the data above we hypothesise that the observed viscosity increase during irradiation of $\mathbf{1}$ is due to the oxidation of unsaturated lipids with reactive oxygen species (ROS), more specifically singlet molecular oxygen. In spite of the fact that $\mathbf{1}$ does not fully integrate into the lipid monolayer, an effective oxidation was shown to occur. This interpretation is consistent with no viscosity increase observed in saturated DPhPC lipid or in the presence of $\mathrm{NaN}_{3}$.

Previously we demonstrated that a porphyrin dimer 2 , structurally similar to $\mathbf{1}$, reported a dramatic increase in viscosity during photoinduced cell death, from 50 to $300 \mathrm{cP}$. Here we set out to test if irradiation of $\mathbf{1}$ and ensuing PDT produces a similar change in viscosity in cells. 1 was incorporated into SK-OV-3 cells, and the microviscosity was imaged by the ratiometric method, which allows rapid detection of viscosity changes. The results are shown in Figure 6 and demonstrate a 3 -fold increase in viscosity in cell cytoplasm upon irradiation, from $5 \mathrm{cP}$ to $13 \mathrm{cP}$. While the viscosities reported are lower the trend remains the same as for $\mathbf{2}$. Due to a low signal and fast bleaching of the dye, viscosity estimation from FLIM images was not possible.
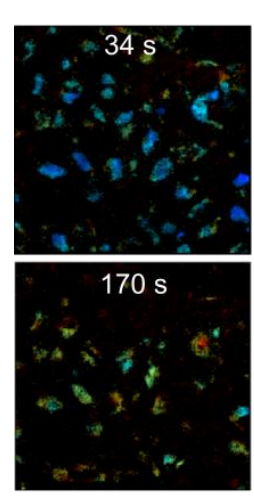

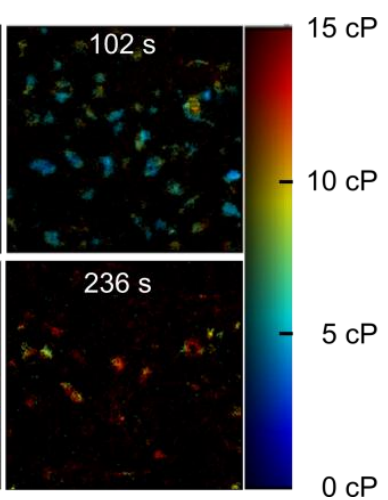

$0 \mathrm{cP}$
Figure 6. Viscosity maps obtained from ratiometric images of SK-OV-3 cells upon different irradiation times, shown in white above images; $\lambda_{\text {exc }}=453 \mathrm{~nm}$.

\section{Conclusions}

In conclusion, we have characterised a red-emitting molecular rotor that works in both the lifetime and the ratiometric measurement modes. We have incorporated the rotor in a range of lipid-based systems and in live cells. We have demonstrated that the combination of both modes allows both quantitative measurements of viscosity and dynamic information to be obtained.

\section{Acknowledgements}

AV thanks the EPSRC for the Prize Studentship. MKK is thankful to the EPSRC for the Career Acceleration Fellowship. This work was partially supported by the European Commission in the form of a Marie Curie individual Fellowship to M.B. under the contract MEIT-CT-2006-041522

\section{Notes and references}

${ }^{a}$ Chemistry Department, Imperial College London, Exhibition Road, SW7 $2 A Z, U K$

${ }^{b}$ University of Oxford, Department of Chemistry, Chemistry Research Laboratory, Oxford, OX1 3TA, UK

$\neq$ Present address: Department of Chemistry, University of Wyoming, Laramie, WY, USA.

$\dagger$ Electronic Supplementary Information (ESI) available: See DOI: $10.1039 / \mathrm{c} 000000 \mathrm{x} /$

(1) Chauvière, A.; Preziosi, L. Cell Mechanics: From Single Scale-Based Models to Multiscale Modeling (Chapman \& Hall/CRC Mathematical \& Computational Biology); Chapman and Hall/CRC, 2010.

(2) Hosny, N. A.; Fitzgerald, C.; Tong, C.; Kalberer, M.; Kuimova, M. K.; Pope, F. D. Faraday Discuss. 2013, 165, 343-356.

(3) Nölle, J. M.; Jüngst, C.; Zumbusch, A.; Wöll, D. Polym. Chem. 2014, $5,2700-2703$.

(4) Hungerford, G.; Allison, A.; McLoskey, D.; Kuimova, M. K.; Yahioglu, G.; Suhling, K. J. Phys. Chem. B 2009, 113, 12067-12074.

(5) Schwille, P. Cell Biochem. Biophys. 2001, 34, 383-408.

(6) Magde, D.; Elson, E.; Webb, W. W. Phys. Rev. Lett. 1972, 29, 705708.

(7) Swaminathan, R.; Hoang, C. P.; Verkman, A. S. Biophys. J. 1997, 72, 1900-1907.

(8) Poo, M.-M.; Cone, R. A. Nature 1974, 247, 438-441.

(9) Kaji, N.; Ogawa, R.; Oki, A.; Horiike, Y.; Tokeshi, M.; Baba, Y. Anal. Bioanal. Chem. 2006, 386, 759-764. 
(10) Levi, V.; Gratton, E. Cell Biochem. Biophys. 2007, 48, 1-15.

(11) Dix, J. A; Verkman, A. S. Biophys. J. 1990, 57, 231-240.

(12) Siegel, J.; Suhling, K.; Lévêque-Fort, S.; Webb, S. E. D.; Davis, D. M.; Phillips, D.; Sabharwal, Y.; French, P. M. W. Rev. Sci. Instrum. 2003, 74, 182-192.

(13) Fushimi, K.; Verkman, A. S. J. Cell Biol. 1991, 112, 719-725.

(14) Kuimova, M. K. Phys. Chem. Chem. Phys. 2012, 14, 12671-12686.

(15) Haidekker, M. A.; Nipper, M.; Mustafic, A.; Lichlyter, D.; Dakanali, M.; Theodorakis, E. A. Dyes with Segmental Mobility: Molecular Rotors; Demchenko, A. P., Ed.; Springer Series on Fluorescence; Springer Berlin Heidelberg: Berlin, Heidelberg, 2010; Vol. 8, pp. 267-308.

(16) Kuimova, M. K.; Yahioglu, G.; Levitt, J. A.; Suhling, K. J. Am. Chem. Soc. 2008, 130, 6672-6673.

(17) Kuimova, M. K.; Botchway, S. W.; Parker, A. W.; Balaz, M.; Collins, H. A.; Anderson, H. L.; Suhling, K.; Ogilby, P. R. Nat. Chem. 2009, 1, 69-73.

(18) Peng, X. J.; Yang, Z. G.; Wang, J. Y.; Fan, J. L.; He, Y. X.; Song, F. L.; Wang, B. S.; Sun, S. G.; Qu, J. L.; Qi, J.; Yang, M. J. Am. Chem. Soc. 2011, 133, 6626-6635.

(19) Haidekker, M. A; Brady, T. P.; Lichlyter, D.; Theodorakis, E. A. J. Am. Chem. Soc. 2006, 128, 398-399.

(20) Haidekker, M. A; Ling, T.; Anglo, M.; Stevens, H. Y.; Frangos, J. A; Theodorakis, E. A. Chem. Biol. 2001, 8, 123-131.

(21) Balaz, M.; Collins, H. A.; Dahlstedt, E.; Anderson, H. L. Org. Biomol. Chem. 2009, 7, 874-888.

(22) Yang, Z.; He, Y.; Lee, J.-H.; Park, N.; Suh, M.; Chae, W.-S.; Cao, J.; Peng, X.; Jung, H.; Kang, C.; Kim, J. S. J. Am. Chem. Soc. 2013, 135, 9181-9185.

(23) Jiang, N.; Fan, J.; Zhang, S.; Wu, T.; Wang, J.; Gao, P.; Qu, J.; Zhou, F.; Peng, X. Sensors Actuators B Chem. 2014, 190, 685-693.

(24) Holden, M. A; Needham, D.; Bayley, H. J. Am. Chem. Soc. 2007, $129,8650-8655$.

(25) Angelova, M. I.; Dimitrov, D. S. 1986, 303-311.

(26) Warren, S. C.; Margineanu, A.; Alibhai, D.; Kelly, D. J.; Talbot, C.; Alexandrov, Y.; Munro, I.; Katan, M.; Dunsby, C.; French, P. M. W. PLoS One 2013, 8, e70687.

(27) Winters, M. U.; Karnbratt, J.; Eng, M.; Wilson, C. J.; Anderson, H. L.; Albinsson, B. J. Phys. Chem. C 2007, 111, 7192-7199.

(28) Kuimova, M. K.; Collins, H. A.; Balaz, M.; Dahlstedt, E.; Levitt, J. A.; Sergent, N.; Suhling, K.; Drobizhev, M.; Makarov, N. S.; Rebane, A.; Anderson, H. L.; Phillips, D. Org. Biomol. Chem. 2009, 7, 889896.

(29) Kuimova, M. K.; Balaz, M.; Anderson, H. L.; Ogilby, P. R. J. Am. Chem. Soc. 2009, 131, 7948-7949.

(30) Forster, T.; Hoffmann, G. Zeitschrift Fur Phys. Chemie-Frankfurt 1971, 75, 63-76.

(31) Wilkinson, J. D.; Wicks, G.; Nowak-Król, A.; Łukasiewicz, Ł. G.; Wilson, C. J.; Drobizhev, M.; Rebane, A.; Gryko, D. T.; Anderson, H. L. J. Mater. Chem. C 2014, 2, 6802.

(32) Lindsey, H.; Petersen, N. O.; Chan, S. I. Biochim. Biophys. Acta 1979, 555, 147-167.

(33) Wu, Y.; Stefl, M.; Olzyńska, A.; Hof, M.; Yahioglu, G.; Yip, P.; Casey, D. R.; Ces, O.; Humpolíčková, J.; Kuimova, M. K. Phys. Chem. Chem. Phys. 2013, 15, 14986-14993.
(34) Yeagle, P. L. The Structure of Biological Membranes, Second Edition; CRC Press, 2004. 